DOI: $10.17516 / 1998-2836-0263$

УДК 541.138: 547.484 .451

\title{
Electrocatalytic Conversion \\ of Levulinic Acid in Acid Medium
}

\author{
Tatyana A. Kenova*a, Nikolay A. Zos'koa, \\ Valentin V. Sychev ${ }^{\mathrm{a}}$ and Oxana P. Taran ${ }^{\mathrm{a}, \mathrm{b}}$ \\ anstitute of Chemistry and Chemical Technology SB RAS \\ Krasnoyarsk, Russian Federation \\ ${ }^{b}$ Siberian Federal University \\ Krasnoyarsk, Russian Federation
}

Received 29.10.2021, received in revised form 07.11.2021, accepted 29.11.2021

\begin{abstract}
The electrochemical hydrogenation of levulinic acid in $\mathrm{H}_{2} \mathrm{SO}_{4}$ solution at aluminium, lead, graphite and glassy carbon electrodes is studied. The process is identified to proceed selectively to valeric acid. The conversion, selectivity and faradaic efficiency are significantly influenced by the material electrode nature. The levulinic acid hydrogenation at glassy carbon is shown for the first time to proceed to valeric acid, and the process selectivity is affected by the concentration of surface functionalities.
\end{abstract}

Keywords: levulinic acid, electrocatalytic hydrogenation, valeric acid, voltammetry, conversion effectivity, process selectivity, preparative electrolysis.

Acknowledgment. The work has been realized within the frame work of the state task Institute Chemistry and Chemical Technology SB RAS (a project 0287-2021-0012) with the equipment of Krasnoyarsk Regional Center for Collective Using FRC KSC SB PAS.

Citation: Kenova, T. A., Zos'ko, N.A., Sychev, V.V., Taran, O. P. Electrocatalytic conversion of levulinic acid in acid medium, J. Sib. Fed. Univ. Chem., 2021, 14(4), 561-569. DOI: 10.17516/1998-2836-0263

(C) Siberian Federal University. All rights reserved

This work is licensed under a Creative Commons Attribution-NonCommercial 4.0 International License (CC BY-NC 4.0).

* Corresponding author E-mail address: kta@icct.ru 


\title{
Электрокаталитическая конверсия \\ левулиновой кислоты в кислой среде
}

\author{
Т.А. Кенова ${ }^{a}$, Н.А. Зосько \\ В. В. Сычев ${ }^{\mathrm{a}}$, О. П. Таран ${ }^{\mathrm{a}, \boldsymbol{\sigma}}$ \\ ${ }^{\mathrm{a}}$ Институт химии и химической технологии \\ ФИЦ КНЦ СО РАН \\ Российская Федерация, Красноярск \\ ${ }^{\sigma}$ Сибирский федеральный университет \\ Российская Федерация, Красноярск
}

\begin{abstract}
Аннотация. Исследован процесс электрохимического гидрирования левулиновой кислоты в водном растворе $\mathrm{H}_{2} \mathrm{SO}_{4}$ на алюминиевом, свинцовом, графитовом и стеклоуглеродном электродах. Установлено, что процесс протекает селективно с образованием валериановой кислоты. Степень конверсии левулиновой кислоты, селективность и фарадеевская эффективность существенно зависят от природы материала электрода. Впервые показано, что на стеклоуглероде гидрирование левулиновой кислоты протекает до валериановой кислоты, причем на селективность процесса оказывает влияние концентрация поверхностных функциональных групп.
\end{abstract}

Ключевые слова: левулиновая кислота, электрокаталитическое гидрирование, валериановая кислота, вольтамперометрия, степень конверсии, селективность процесса, препаративный электролиз.

Благодарность. Работа выполнена в рамках государственного задания Института химии и химической технологии СО РАН (проект 0287-2021-0012) с использованием оборудования Красноярского регионального центра коллективного пользования ФИЦ КНЦ СО РАН.

Цитирование: Кенова, Т.А. Электрокаталитическая конверсия левулиновой кислоты в кислой среде / Т. А. Кенова, Н. А. Зосько, В. В. Сычев, О.П. Таран // Журн. Сиб. федер. ун-та. Химия, 2021, 14(4). С. 561-569. DOI: 10.17516/1998-2836-0263

\section{Введение}

Левулиновая кислота (ЛК), получаемая в результате кислотного каталитического гидролиза растительных полисахаридов, является перспективным сырьем для синтеза продуктов с высокой добавленной стоимостью и производства жидкого биотоплива $[1,2]$. Наличие в молекулярной структуре двух реакционноспособных функциональных групп (кетонной и карбоксильной) делает ЛК важным источником для получения полимеров, фармацевтических препаратов, ароматизаторов, растворителей, пластификаторов [3,4]. Процессы получения указанных соединений, основанные на гомогенном или гетерогенном катализе, исследованы достаточно детально [5-7], тогда как использование электрокаталитических процессов применительно к переработке ЛК изучено слабо [8-10]. Электрохимическая конверсия ЛК до $\gamma$-валеролактона (ГВЛ) в щелочной среде на свинцовом катоде была описана 
еще Тафелем в 1911 г. [11]. Однако в настоящее время имеется ограниченное количество публикаций по электрокаталитическому гидрированию ЛК. В [8] авторы получили валериановую кислоту (ВК) с высокими селективностью (95\%) и фарадеевской эффективностью $(86,5 \%)$ на свинцовом электроде в кислой среде, тогда как при $\mathrm{pH}=7,5$ восстановление идет со 100\%-ным выходом по $\gamma$-валеролактону. Интересный подход предложен авторами $[10,12]$ для получения n-октана через двухстадийный процесс (гидрирование и реакция Кольбе) электрокаталитической конверсии ЛК. Электрокаталитическое гидрирование ЛК является гетерогенным процессом и, следовательно, зависит от природы материала катода. В работе [12] авторы исследовали процесс электрохимической конверсии ЛК на $\mathrm{Pb}, \mathrm{Cu}, \mathrm{Fe}, \mathrm{Ni}$ и графите. Высокие селективность и фарадеевская эффективность в процессе гидрирования ЛК до валериановой были получены на свинце, имеющем наибольшее перенапряжение в реакции выделения $\mathrm{H}_{2}$. Гидрирование ЛК на графите в кислой среде протекает с высокой селективностью до ГВЛ. На других электродах авторы наблюдали низкую эффективность гидрирования ЛК, при этом селективность продуктов зависела от $\mathrm{pH}$ среды. Высокие показатели процесса гидрирования ЛК на свинце не делают его, однако, достойным кандидатом при использовании в промышленных масштабах из-за опасности попадания высокотоксичных $\mathrm{Pb}^{2+}$-ионов в сточные воды. В связи с этим поиск и исследование новых материалов для процесса электрокаталитического гидрирования ЛК выступают на сегодняшний день актуальной задачей.

В данной работе представлены результаты исследования электрокаталитических свойств алюминия (Al), стеклоуглерода (СУ), графита в сравнении со свинцом (Pb) в процессе гидрирования левулиновой кислоты до валериановой в кислой среде.

\section{Экспериментальная часть}

Исследования выполняли в растворах с концентрацией 0,2 моль/л левулиновой кислоты (SigmaAldrich) в $0,5 \mathrm{M} \mathrm{H}_{2} \mathrm{SO}_{4}$ (хч), $\mathrm{pH}=0,2$, приготовленных с использованием деионизированной воды (18,2 МОм), полученной на установке Simplicity (Millipore Corp., France).

Эксперименты по гидрированию ЛК проводили в электрохимической трехэлектродной ячейке с разделением катодного и анодного пространства катионообменной мембраной МФ-4СК на электродах из свинца, алюминия стеклоуглерода (СУ), графита. Раствор в католитную камеру подавали при помощи перистальтического насоса LeadFluidBT300L (LeadFluidTech., China) и циркулировали со скоростью 80 мл/мин.

Площадь катода составляла 5 см², объем электролита - 40 мл, вспомогательным электродом служила платиновая фольга. В качестве электрода сравнения использовали насыщенный хлорсеребряный электрод (ХСЭ) (+0,195 В отн. СВЭ), соединенный солевым мостиком с капилляром Луггина. Все значения потенциалов в тексте статьи указаны относительно данного электрода сравнения.

Гидрирование ЛК проводили в потенциостатических условиях при значении потенциала от $-1,2$ до -1,8 В, продолжительность электролиза составляла 1-3 ч. Перед регистрацией вольтамперных зависимостей растворы продували аргоном в течение 30 мин для удаления растворенного кислорода. Потенциал электрода поддерживали при помощи потенциостата / гальваностата / FRA BIOLOGIC VSP-300 (Bio-Logic SAS, Clarix, France).

$$
-563-
$$


Перед началом эксперимента для каждого электрода проводили катодную активацию сканированием потенциала на протяжении 20 циклов со скоростью $100 \mathrm{MB} / \mathrm{c}$ от равновесного потенциала соответствующего электрода до -1,6 В.

Продукты гидрирования ЛК были проанализированы методом ВЭЖХ на приборе Милихром А-02 («ЭкоНова», Россия), оснащенном УФ-детектором (регистрация при $\lambda=210$ нм) и хроматографической колонкой «Диасфер-250-ПА», 5 мкм, 2×75 мм («ЭкоНова», Россия). В качестве элюента использовали $85 \% 0.075 \mathrm{M} \mathrm{LiClO}_{4}, 15 \% \mathrm{CAN} \mathrm{H}_{2} \mathrm{O}$, подаваемый со скоростью 0,2 мл/мин при температуре $40{ }^{\circ} \mathrm{C}$.

Степень конверсии левулиновой кислоты, выход и селективность по отношению к ВК рассчитывали согласно уравнениям (1)-(3):

$$
\begin{aligned}
\chi_{Л К} & =\frac{c_{Л К, 0}-c_{Л К}}{c_{Л К, 0}} \cdot 100 \%, \\
Y_{\mathrm{BA}} & =\frac{c_{\mathrm{BA}}}{c_{Л К, 0}} \cdot 100 \%, \\
S_{\mathrm{BA}} & =\frac{Y_{\mathrm{BA}}}{\chi_{л К}} \cdot 100 \%,
\end{aligned}
$$

где $\chi_{\text {лк }}-$ конверсия ЛК, мол.\%; $\mathrm{C}_{\text {Лк,0}}-$ начальная концентрация ЛК моль/л; $\mathrm{C}_{\text {лк }}-$ текущая концентрация ЛК моль/л; $\mathrm{Y}_{\text {ВК }}$ выход ВК мол.\%; $\mathrm{S}_{\text {ВК }}$ - селективность по отношению к ВК\%.

Фарадеевскую эффективность рассчитывали по формуле

$$
C E=\frac{n_{Л \kappa} z F}{I \tau},
$$

где $n_{\text {лк }}$ - количество молей левулиновой кислоты, моль; $z$ - число электронов, участвующих в реакции (для гидрирования ЛК до ВК, $z=4$ ); $F$ - число Фарадея, 26,8 А ч; I - ток электролиза, А; $\tau$ - время электролиза, ч.

\section{Результаты и обсуждение}

Скорость и селективность электрохимического процесса в значительной степени определяются природой электродного материала, поэтому при выборе материала катода для процесса гидрирования ЛК важно оценить его электрокаталитическую активность как по отношению к самому субстрату, так и в реакции выделения водорода.

С термодинамической точки зрения гидрирование ЛК до ВК является благоприятным процессом, так как обратимый потенциал восстановления ЛК до ВК приблизительно на 500 мВ положительнее, чем обратимый потенциал реакции выделения водорода (ОВЭ) во всем диапазоне $\mathrm{pH}$. Поскольку реакция восстановление ЛК включает $\mathrm{H}_{2} \mathrm{O}$ и $\mathrm{H}^{+}$, обратимый потенциал изменяется в соответствии с $\mathrm{pH}$ электролита.

В кислой среде восстановление левулиновой кислоты протекает по реакции

$$
\mathrm{CH}_{3} \mathrm{CO}(\mathrm{CH})_{2} \mathrm{COOH}+4 \mathrm{H}^{+}+4 \mathrm{e}^{-} \rightarrow \mathrm{CH}_{3}\left(\mathrm{CH}_{2}\right)_{3} \mathrm{COOH}+\mathrm{H}_{2} \mathrm{O} ;+0,54 \mathrm{~B} \text { (OBЭ). }
$$

Однако кинетически реакция выделения водорода протекает быстрее на металлических электродах по сравнению с реакцией гидрирования ЛК, и, следовательно, действительные потенциалы при электролизе будут достаточно близкими. В зависимости от значения и природы перенапряжения водорода гидрирование органического субстрата на металлах может про- 


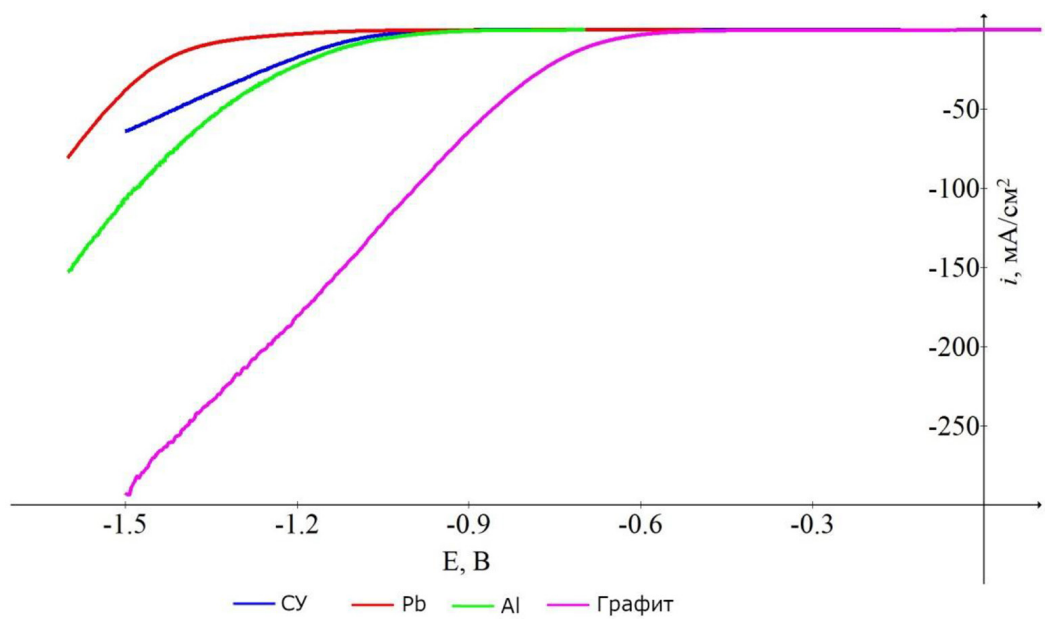

Рис. 1. Вольтамперные потенциодинамические зависимости реакции выделения водорода в $0,5 \mathrm{M} \mathrm{H}_{2} \mathrm{SO}_{4}$ на различных электродных материалах. Скорость развертки потенциала $-50 \mathrm{MB} / \mathrm{c}$

Fig. 1. Linear sweep voltammetry of hydrogen evolution reactionin $0,5 \mathrm{M} \mathrm{H}_{2} \mathrm{SO}_{4}$ at various electrode materials. Ascanrate of $50 \mathrm{mV} / \mathrm{s}$

текать как по электрохимическому (сопряженный протон-электронный перенос заряда), так и по электрокаталитическому механизму, т. е. с участием адсорбированных атомов водорода $[9,13]$.

Вольтамперные зависимости для процесса выделения водорода в 0,5 $\mathrm{M} \mathrm{H}_{2} \mathrm{SO}_{4} \mathrm{Ha} \mathrm{Pb}, \mathrm{Al}$, $\mathrm{CУ}$ и графите, полученные методом линейной развертки потенциала, представлены на рис. 1.

Исследованные электроды различаются как по потенциалу начала выделения (перенапряжению выделения) $\mathrm{H}_{2}$, так и по скорости процесса. Заметное выделение водорода на $\mathrm{Pb}$-катоде наблюдали только при $\mathrm{E}=-1,1 \mathrm{~B}$, для $\mathrm{Al}$ и СУ эти значения составили $-0,88$ и $-0,89 \mathrm{~B}$ соответственно, тогда как на графите было отмечено самое низкое перенапряжение выделения водорода, $-0,32$ В. Различный наклон поляризационных кривых также свидетельствует о разной лимитирующей (скорость определяющей) стадии процесса выделения водорода.

На рис. 2 представлены вольтамперные зависимости процесса электрокаталитического гидрирования 0,2 М ЛК в 0,5 $\mathrm{M} \mathrm{H}_{2} \mathrm{SO}_{4}$ на $\mathrm{Pb}, \mathrm{Al}, \mathrm{CУ}$ и графите.

Анализ полученных вольтамперных зависимостей свидетельствует, что только на свинцовом электроде (рис. $2 a$ ) наблюдается сдвиг потенциала восстановления ЛК в область более низких значений и существенное увеличение плотности тока по сравнению со значениями, полученными для фонового электролита. Так, при потенциале -1,6 В плотность тока составила 81 и $214 \mathrm{~mA} / \mathrm{cm}^{2}$ для реакции выделения водорода и гидрирования ЛК соответственно.

На электроде из СУ (рис. 26) кривые линейной развертки потенциала для фонового электролита и при добавлении ЛК практически совпадают, а плотность тока увеличивается незначительно лишь при потенциалах выше -1,3 В. Увеличение перенапряжения реакции при добавлении в раствор ЛК и, соответственно, снижение плотности тока при одинаковых значениях потенциала по сравнению с фоновым электролитом наблюдается на $\mathrm{Al}$ и графите (рис. 26, г).

Такое поведение, возможно, обусловлено различными характером взаимодействия и энергией связи карбонильной группы в молекуле ЛК с активными центрами на поверхности метал-

$$
-565-
$$




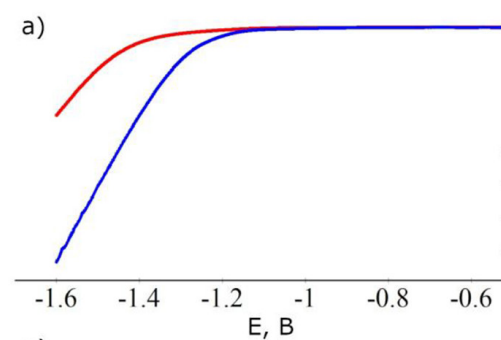

$$
\text { B) }
$$$$
\text { B) }
$$

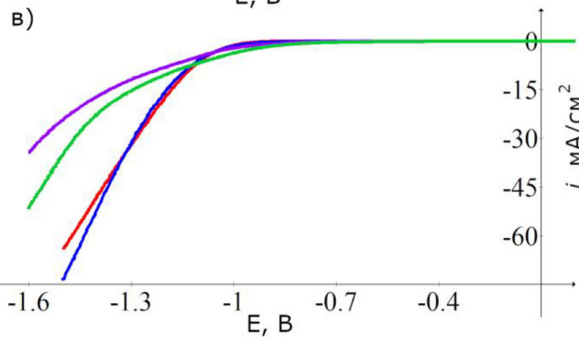

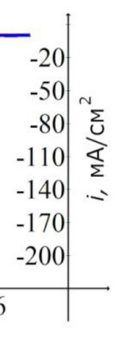

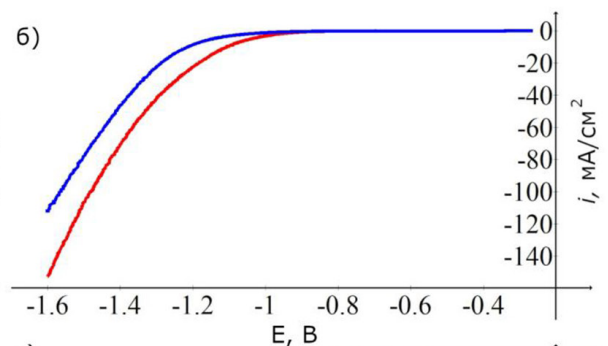

г)

$-0.5 \mathrm{M} \mathrm{H}_{2} \mathrm{SO}_{4}-0.5 \mathrm{M} \mathrm{H}_{2} \mathrm{SO}_{4}+0.2 \mathrm{M} л \mathrm{~K}-0.5 \mathrm{M} \mathrm{H}_{2} \mathrm{SO}_{4}+0.2 \mathrm{M} л \mathrm{~K}$ акт. $-0.5 \mathrm{M} \mathrm{H}_{2} \mathrm{SO}_{4}$ акт.

Рис. 2. Вольтамперные потенциодинамические зависимости реакции выделения водорода и восстановления $0,2 \mathrm{M}$ ЛК в $0,5 \mathrm{M} \mathrm{H}_{2} \mathrm{SO}_{4}$ на различных электродных материалах: $\mathrm{a}-\mathrm{Pb}$; 6 - $\mathrm{Al}$; в - $\mathrm{CV}$; г- графит. Скорость развертки потенциала $50 \mathrm{MB} / \mathrm{c}$

Fig. 2. Linear sweep voltammetry of hydrogen evolution reaction and reduction of $0,2 \mathrm{M} \mathrm{LA}$ in $0,5 \mathrm{M} \mathrm{H}_{2} \mathrm{SO}_{4}$ at various electrode materials: $\mathrm{a}-\mathrm{Pb} ; \mathrm{b}-\mathrm{Al} ; \mathrm{c}-\mathrm{GC}$; $\mathrm{d}-$ graphite. Ascanrate of $50 \mathrm{mV} / \mathrm{s}$

лических и углеродных электродов [14]. Различная адсорбционная способность интермедиатов и продуктов реакции также может оказывать влияние на стадии разряда и рекомбинации ионов водорода и, как следствие, на скорость процесса в целом.

Для оценки влияния природы материала катода на селективность и фарадеевскую эффективность(СЕ) процесса гидрирования был проведен препаративный электролиз при потенциале $-1,6$ В в течение 2 ч. На рис. 3 представлены показатели процесса гидрирования ЛК как функция материала катода.

Как следует из полученной диаграммы, на всех использованных электродах в $0,5 \mathrm{M} \mathrm{H}_{2} \mathrm{SO}_{4}$ электрокаталитическое гидрирование ЛК идет с образованием ВК. Степень конверсии ЛК снижается в ряду $\mathrm{Pb}>\mathrm{CУ}>\mathrm{Al}>$ графит, а селективность возрастает в ряду СУ < графит $<\mathrm{Al}<\mathrm{Pb}$. Исходя из данных вольтамперометрии, основным процессом, протекающим на $\mathrm{Al}$, должно быть выделение $\mathrm{H}_{2}$, тем не менее конверсия ЛК составила более $40 \%$, а селективность по ВК $-38,2$ \%. Al относится к металлам с высоким перенапряжением выделения водорода, и в соответствии с механизмом, предложенным авторами [8], гидрирование $-\mathrm{C}=\mathrm{O}$ в ЛК должно приводить к образованию метильной группы, т. е. селективному образованию ВК. Достаточно низкая селективность Al может свидетельствовать о протекании процесса по иному механизму, например, как предполагается в работе [9], через образование на поверхности электрода радикала 4-гидроксипентановой кислоты, который в зависимости от условий электролиза и природы материала катода либо протонируется (ГВЛ и/или ВК), либо взаимодействует с другим радикалом, образуя димерный продукт (пинакол) (рис. 4).

Углеродные материалы широко используются в качестве катализаторов для электрохимических процессов благодаря таким свойствам, как инертность и (электро)химическая 


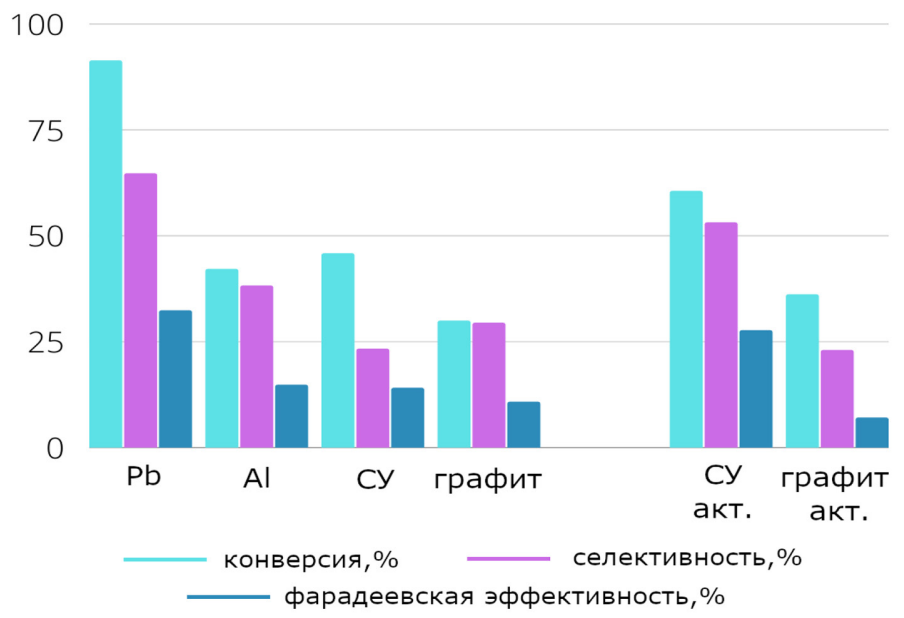

Рис. 3. Электрокаталитическое гидрирование ЛК в $0,5 \mathrm{M} \mathrm{H}_{2} \mathrm{SO}_{4}$ как функция природы материала электрода. Время электролиза 2 ч; потенциал электрода $-1,6 \mathrm{~B}$; начальная концентрация ЛК 0,2 M

Fig. 3. Electrocatalytic hydrogenation of $\mathrm{LA}$ in $0.5 \mathrm{M} \mathrm{H}_{2} \mathrm{SO}_{4}$ as a function of the nature of electrode material. The electrolysis duration $2 \mathrm{~h}$; an electrode potential of $-1,6 \mathrm{~V}$; the initial LA concentration $0.2 \mathrm{M}$

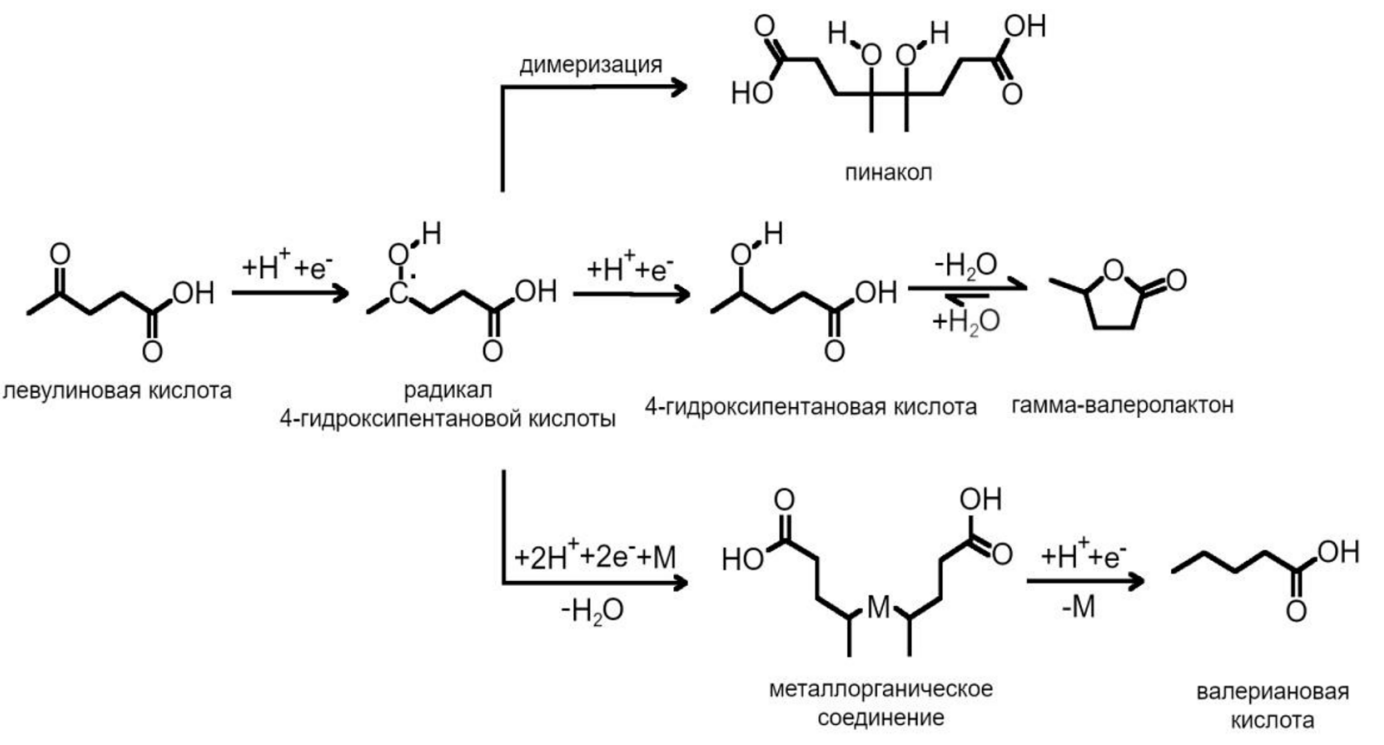

Рис. 4. Адаптированный для ЛК механизм восстановления до ВК, ГВЛ и пинакола, основанный на общем механизме восстановления карбонильной группы [9]

Fig. 4. Adapted reduction mechanism for LA to VA, gVL and pinacol based on the generic carbonyl reduction mechanism [9]

стойкость. С другой стороны, на поверхности углеродных материалов можно синтезировать различные функциональные кислородсодержащие группы, которые выступают в качестве активных центров адсорбции реагентов и продуктов реакции. СУ часто используется как сенсорный электрод и для исследования механизмов различных электрохимических реакций. Используя катодную и/или анодную обработку СУ, можно изменять природу и концентрацию активных центров на его поверхности. В этой связи исследование СУ в качестве катализато- 
ра для восстановления ЛК, а также возможности его активации представляет теоретический и практический интерес.

В данной работе активацию СУ электрода проводили в $0,5 \mathrm{M} \mathrm{H}_{2} \mathrm{SO}_{4}$ и $1 \mathrm{M} \mathrm{KOH} \mathrm{при} \mathrm{потен-}$ циалах выделения водорода $-1,5$ В и кислорода 2,3 В в течение 600 с по методике, описанной в [15]. После обработки степень конверсии ЛК увеличилась до 60,5 \%, а селективность и СЕ составили 53,1 и 27,7 \% соответственно (рис. 3). Кроме того, после обработки СУ изменился также ход вольтамперных зависимостей (см. рис. 28), что связано, по-видимому, с изменением количества функциональных групп на поверхности электрода [16].

Обработка графита, однако, не приводит к заметному увеличению конверсии ЛК, при этом селективность по отношению к ВК и фарадеевская эффективность снижаются (рис. 3).

При одинаковых условиях электролиза лучшие результаты были получены на металлическом свинце. Степень конверсии ЛК за 2 ч электролиза составила 91,3 \% при селективности по отношению к ВК 64,7 \% и фарадеевской эффективности 32,4%. Следует отметить, что в данной работе селективность Рb по отношению к ВК ниже, чем в работах $[4,9,11]$. Такое расхождение в показателях процесса, на наш взгляд, определяется существенным различием фиксируемых плотностей тока (при аналогичных других параметрах электролиза). Так, в работах $[4,9]$ плотность тока исходя из представленных вольтамперных зависимостей составляла от 0,4 до 40 мА/см² при потенциале $-1,6$ В, тогда как в настоящей работе она более 200 $\mathrm{MA} / \mathrm{cm}^{2}$ (см. рис. $2 a$ ). Можно предположить, что в данном случае существует отличие в фактической площади электродов, вероятно, из-за способа активации его поверхности. Высокие плотности тока могут приводить к изменению рН в приэлектродном слое и потенциально влиять на адсорбционные характеристики ЛК, приводя к иному распределению продуктов.

\section{Заключение}

Электрокаталитическое гидрирование левулиновой кислоты в водном растворе $\mathrm{H}_{2} \mathrm{SO}_{4}$ было исследовано на алюминиевом, свинцовом, графитовом и стеклоуглеродном электродах. Установлено, что на всех исследованных электродах электрокаталитическое гидрирование ЛК протекает до валериановой кислоты. Показано, что степень конверсии ЛК снижается в ряду $\mathrm{Pb}>\mathrm{CV}>\mathrm{Al}>$ графит, а селективность возрастает в ряду СУ $<$ графит $<\mathrm{Al}<\mathrm{Pb}$. Впервые показано, что на стеклоуглероде гидрирование ЛК протекает до ВК, причем на селективность процесса оказывает влияние состояние поверхности СУ.

\section{Конфликт интересов}

Авторы заявляют, что у них нет конфликта интересов.

\section{Список литературы / References}

1. Malu T. J., Manikandan K., Cheralathan K.K. Chapter 6 - Levulinic acid - a potential keto acid for producing biofuels and chemicals. Biomass, Biofuels, Biochemicals, Recent Advances in Development of Platform Chemicals 2020, P. 171-197.

2. Taran O.P., Sychev V.V., Kuznetsov B.N. $\gamma$-Valerolactone as a Promising Solvent and Basic Chemical Product: Catalytic Synthesis from Plant Biomass Components. Catalysis in Industry, 2021, Vol. 13, No. 3, P. 289-308. 
3. Taran O.P., Gromov N.V., Parmon V.N. Chapter 2 - Catalytic Processes and Catalyst Development in Biorefining, Green Chemistry Series, Sustainable Catalysis for Biorefineries 2018, No. 56, P. 25-64

4. Du Y., Chen X., Qi J., Wang P., Liang C. Synthesis of Valeric Acid by Selective Electrocatalytic Hydrogenation of Biomass-Derived Levulinic Acid. Catalysts 2020, Vol. 10, P. 692-703.

5. Carnevali D., Rigamonti M.G., Tabanelli T., Patience G. S., Cavani F. Levulinic acid upgrade to succinic acid with hydrogen peroxide. Applied Catalysis A, General 2018, Vol. 563, P. 98-104.

6. S. Dutta, Yu I. K.M., Tsang D. C.W., Ng Y.H., Ok Y. S., Sherwood J., Clark J. H. Green synthesis of gamma-valerolactone (GVL) through hydrogenation ofbiomass-derivedlevulinic acid using nonnoble metal catalysts: A criticalreview. Chemical Engineering Journal 2019, Vol. 372, P. 992-1006.

7. Yan L., Yao Q., Fu Y. Conversion of levulinic acid and alkyl levulinates into biofuelsand highvalue chemicals, Green Chemisry 2017, Vol.19, P. 5527-5547.

8. Xin L., Zhang Z., Qi J., Chadderdon D. J., Qiu Y., Warsko K. M., Li W. Electricity Storage in Biofuels: Selective ElectrocatalyticReduction of Levulinic Acid to Valeric Acid or g-Valerolactone. ChemSusChem 2013, Vol. 6, P. 674-686.

9. Bisselink R.J. M., Crockatt M., Zijlstra M., Bakker I.J., Goetheer E., Slaghek T.M., van Es D.S. Identification of More Benign Cathode Materials for the Electrochemical Reduction of Levulinic Acid to Valeric Acid. Chem Electro Chem 2019, Vol. 6, P. 3285-3290.

10. Nilges P., dos Santos T. R., Harnisch F., Schröder U. Electrochemistry for biofuel generation: Electrochemical conversion oflevulinic acid tooctane. Energy Environmental Science 2012, Vol. 5, P. 5231-5235.

11.TafelJ.,EmmertB. DieelektrolytischeReduktionvonLävulinsäureund $\alpha$-Dimethyllävulinsäure. Zeitschrift für Electrochemie1911, Vol. 17, P. 569-572.

12. dos Santos T.R., Nilges P., Sauter W., Harnisch F., Schröder U. Electrochemistry for the generation of renewablechemicals: electrochemical conversion oflevulinicacid. RSC Advances 2015, Vol. 5, P. 26634-26643.

13. Koh K., Sanyal U., Lee M.-S., Cheng G., Song M., Glezakou V.-A., Liu Y., Li D., Rousseau R., Gutiérrez O. Y., Karkamkar A., Derewinski M., Lercher J.A. Electrochemically Tunable ProtonCoupled Electron Transfer in Pd-Catalyzed Benzaldehyde Hydrogenation. Angewandte. Chemie 2020, Vol. 132, P. 1517-1521.

14. Song Y., Sanyal U., Pangotra D., Holladay J.D., Camaioni D.M., Gutiérrez O.Y., Lercher J.A. Hydrogenation of benzaldehyde via electrocatalysis and thermalcatalysis on carbonsupported metals. Journal of Catalysis 2018, Vol. 359, P. 68-75.

15. Dekanski A., Stevanović J., Stevanović R., Nikolić B.Ź., Jovanović V.M. Glassy carbon electrodes. I. Characterization and electrochemical activation. Carbon 2001, Vol. 39, P. 1195-1205.

16. Bystron T., Sramkova E., Dvorak F., Bouzek K. Glassy carbon electrode activation e A way towards highly active, reproducible and stable electrode surface. Electrochimica Acta 2019, Vol. 299, P. 963-970. 